\title{
Errata for: Locally Computable UOWHF with Linear Shrinkage
}

\author{
Benny Applebaum* $\quad$ Yoni Moses ${ }^{\dagger}$
}

This is an errata for our Journal of Cryptology paper, "Locally Computable UOWHF with Linear Shrinkage" [2]. There is a gap in the proof of Theorem 4.1 that asserts that the collection $\mathcal{F}_{P, n, m}$ is $\delta$-secure $\beta$-random target-collision resistant assuming the one-wayness and the pseudorandomness of the collection for related parameters. We currently do not know whether Theorem 4.1 (as stated in Section 4) holds.

The source of trouble is a miscalculation in the proof of Claim 4.4. Indeed, it is essentially claimed that for a random graph $G$ and random input $x \in\{0,1\}^{n}$, any string $z \in\{0,1\}^{n}$ whose output $f_{G, P}(z) \in\{0,1\}^{2 m}$ agrees with $f_{G, P}(x) \in\{0,1\}^{2 m}$ on about $(1+\gamma) m$ locations, must be correlated with $x$. Unfortunately, this level of "output correlation" is not significant enough to guarantee the desired input correlation.

We note that Theorem 5.1 that transforms any $\delta$-secure $\beta$-random target collision resistant collection to a target collision resistant collection while preserving constant locality and linear shrinkage, remains intact. Thus, one can construct a locally computable UOWHF with linear shrinkage based on the hypothesis that random local functions are $\delta$-secure $\beta$-random target-collision resistant. Specifically, the main result of the paper can be based (via Theorem 5.1) on the following hypothesis.

Assumption 1. For every constants $\varepsilon, \beta, \delta>0$, there exists an integer $d$ and a $d$-local predicate $P:\{0,1\}^{d} \rightarrow\{0,1\}$ such that the ensemble $\mathcal{F}_{P, n,(1-\varepsilon) n}$ is o(1)-secure $\beta$-random target-collision resistance, that is, every polynomial-time adversary $\mathcal{A}$ that is given a random local function $f \stackrel{R}{\leftarrow}$ $\mathcal{F}_{P, n,(1-\varepsilon) n}$ and a random target $x \stackrel{R}{\leftarrow}\{0,1\}^{n}$, outputs $x^{\prime} \in f^{-1}(f(x))$ which is $\beta n$-far from $x$ with probability at most $\delta$.

In fact, the assumption seems plausible even for $\delta=o(1)$. We also mention that locallycomputable functions with linear-shrinkage that achieve a stronger form of collision-resistance were constructed in [1] based on incomparable assumptions.

Acknowledgement. We are grateful to Colin Sandon for pointing out the gap in Claim 4.4.

\section{References}

[1] B. Applebaum, N. Haramaty, Y. Ishai, E. Kushilevitz, and V. Vaikuntanathan. Low-complexity cryptographic hash functions. In C. H. Papadimitriou, editor, 8th Innovations in Theoretical

\footnotetext{
${ }^{*}$ School of Electrical Engineering, Tel-Aviv University, bennyap@post.tau.ac.il.

${ }^{\dagger}$ ymoses@gmail.com.
} 
Computer Science Conference, ITCS 2017, January 9-11, 2017, Berkeley, CA, USA, volume 67 of LIPIcs, pages 7:1-7:31. Schloss Dagstuhl - Leibniz-Zentrum für Informatik, 2017.

[2] B. Applebaum and Y. Moses. Locally computable UOWHF with linear shrinkage. J. Cryptol., 30(3):672-698, 2017. 